\title{
Études climatologiques.
}

Par

\section{DEZYDERY SZYMKIEWICZ.}

\section{Sur une nouvelle forme de l'actinomètre blanc-noir.}

J'ai décrit, dans la partie $X$ de ces études ${ }^{1}$ ), un simple dispositif permettant de mesurer la radiation de longueur d'onde moindre que $3 \mu$. C'étaient tout simplement deux thermomètres, l'un blanchi, la'utre noirci, montés dans une petite caisse en bois. L'appareil réagissait bien à l'action du rayonnement, mais il était sensible à l'action du vent. Je me suis gardé de le recouvrir de verre, pour ne pas provoquer un échauffement continuel.

Heureusement, j'ai pu me persuader que cet échauffement des thermomètres qui se produit sous le verre n'empêche pas de mesurer la radiation.

J'ai construit deux nouveaux types de l'actinomètre en question. L'un d'eux ne différait du modèle primitif que par la plaque en verre qui recouvrait la caisse, ou plus exactement le compartiment de cette caisse contenant les réservoirs des thermomètres. Dans le second type, les thermomètres ont été protégés contre l'action du vent par les parois d'un vase sphérique. Dans le col de ce vase, les thermomètres ont été fixés au moyen d'un bouchon (fig. 89). Le vase est d'en bas barbouillé de gypse pour régulariser la reflexion de la radiation. Le tout est monté dans un dispositif en bois, permettant d'orienter l'appareil.

Le réservoir d'un des thermomètres de l'appareil est noirci dans la fumée de l'essence de térébenthine. Le second thermomètre est blanchi dans la fumée se dégageant pendant la combustion du magnésium. L'épaisseur de l'enduit noir et blanc produit une influence $60-63$.

1) „Sur un nouvel actinomètre“. - Acta Soc. Bot. Polon. Vol. IV (1926), 
considérable sur l'échauffement des thermomètres. On doit donc conserver l'enduit une fois appliqué. Le noir est très stable. Au contraire, le blanc, composé de l'oxyde de magnésium, est très sensible à l'action de l'humidité. Pour le conserver intact, il faut munir l'appareil d'un tube à chlorure de calcium, ce qu'on peut voir sur la fig. 89 .

Les deux types de l'actinomètre fonctionnent également bien. Le second n'est préférable que par la facilité qu'il présente pour garantir les thermomètres de l'humidité.

Comme j'ai déjà insisté dans la partie $\mathrm{X}$ de ces études, l'actinomètre dont il est question ici ne mesure que l'intensité maximum du rayonnement tombant à un endroit donné. La cause en est la rondeur des récipients des thermomètres. Avec les récipients aplatis, il serait peut-être possible de mesurer la radiation tombant à un plan déterminé, par exemple sur un plan horizontal.

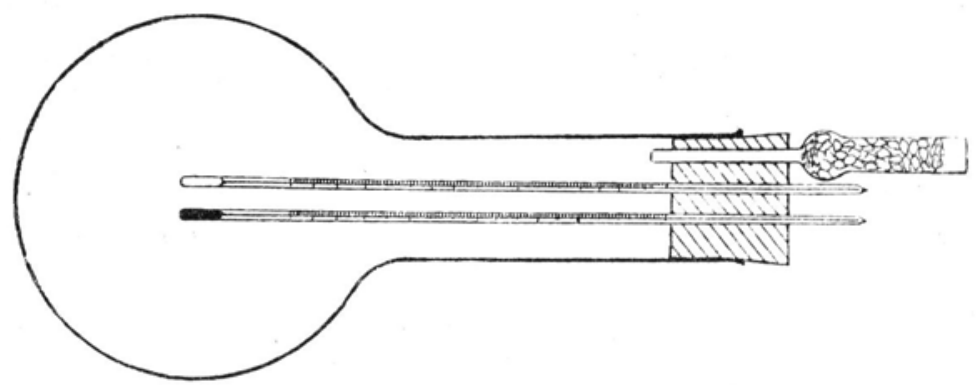

Ryć. 89.

L'échauffement continuel, provoqué à soleil par l'action de l'enveloppe en verre, se produit de telle façon que d'abord le thermomètre noir s'échauffe plus vite que le blanc, mais à partir d'un certain moment, après 5 minutes environ, les deux thermomètres montent parallèlement. Par exemple, on a obtenu par un jour clair les valeurs suivantes, notées toutes les minutes (table I).

Il s'ensuit que la mesure de la radiation au moyen des.appareils de ce genre exige l'invariabilité de la radiation pendant 5 minutes au moins.

Étalonnage des appareils, exécuté dans la Station Écologique de la Faculté Agricole et Forestière de l'École Polytechnique de Lwów à Dublany, au moyen d'un pyranomètre système Anders Angström, a montré que la différence de la température entre les thermomètres blanc et noir est proportionnelle à l'intensité de la radiation. On peut citer comme exemple les mesures exécutées par 
Table I.

\begin{tabular}{|c|c|c|}
\hline \multicolumn{2}{|c|}{ Thermom ètres } & \multirow{2}{*}{ Différence } \\
\hline Noir & Blanc & \\
\hline 50.0 & $38 \cdot 0$. & $12 \cdot 0$ \\
\hline $51 \cdot 6$ & $39 \cdot 2$ & $12 \cdot 4$ \\
\hline $52 \cdot 6$ & $40 \cdot 1$ & $12 \cdot 5$ \\
\hline $53 \cdot 2$ & $40 \cdot 6$ & 126 \\
\hline $54 \cdot 1$ & $41 \cdot 4$ & $12 \cdot 7$ \\
\hline $55^{\prime} 1$ & $42 \cdot 4$ & $12 \cdot 7$ \\
\hline $55 \cdot 7$ & 43.0 & $12 \cdot 7$ \\
\hline $56 \cdot 2$ & $43 \cdot 5$ & $12 \cdot 7$ \\
\hline $56 \cdot 7$ & $44 \cdot 0$ & $12 \cdot 7$ \\
\hline $57 \cdot 0$ & $44 \cdot 3$ & $12 \cdot 7$ \\
\hline $57 \cdot 1$ & $44 \cdot 4$ & $12 \cdot 7$ \\
\hline $57 \cdot 6$ & 44.9 & $12 \cdot 7$ \\
\hline $57 \times 7$ & 450 & $12 \cdot 7$ \\
\hline
\end{tabular}

Table II.

\begin{tabular}{|c|c|c|c|c|}
\hline \multirow{2}{*}{$\begin{array}{c}\text { Radiation } \\
\text { en } \\
\text { gr. cal./min. } \mathrm{cm}^{2}\end{array}$} & \multicolumn{2}{|c|}{ Thermom ètres } & \multirow{2}{*}{ Différence } & \multirow{2}{*}{$\begin{array}{c}\text { Température } \\
\text { de l'air }\end{array}$} \\
\hline & Noir & Blanc & & \\
\hline 1.86 & $56 \cdot 8$ & $36 \cdot 4$ & 20.4 & $24 \cdot 0$ \\
\hline $1 \cdot 83$ & $57 \cdot 8$ & $38 \cdot 3$ & 19.5 & $24^{\circ} 0$ \\
\hline 1.72 & $57 \cdot 0$ & 38.0 & $19 \cdot 0$ & $23 \cdot 8$ \\
\hline $1 \cdot 27$ & 50.6 & $35 \cdot 6$ & $15^{\circ} 0$ & $22 \cdot 8$ \\
\hline $1 \cdot 21$ & $49 \cdot 6$ & 350 & $14 \cdot 6$ & $22 \cdot 6$ \\
\hline $1 \cdot 16$ & $48 \cdot 6$ & $34 \cdot 6$ & 14.0 & $22 \cdot 4$ \\
\hline 0.93 & $44 \cdot 6$ & 33.0 & $11 \cdot 6$ & $22 \cdot 4$ \\
\hline 0.90 & $43 \cdot 6$ & $32 \cdot 3$ & $11 \cdot 3$ & $22 \cdot 1$ \\
\hline 0.85 & $41 \cdot 6$ & 30.8 & $10 \cdot 8$ & $22 \cdot 1$ \\
\hline 0.77 & $40 \div 6$ & 30.5 & $10 \cdot 1$ & $21 \cdot 7$ \\
\hline 0.73 & $39 \cdot 4$ & $30^{\circ} 0$ & $9 \cdot 4$ & $21 \cdot 2$ \\
\hline 0.64 & $37 \cdot 9$ & $29 \cdot 0$ & $8 \cdot 9$ & $21^{\circ} 0$ \\
\hline 0.54 & $36 \cdot 3$ & $28 \cdot 2$ & $8 \cdot 1$ & $20 \cdot 8$ \\
\hline 0.47 & $33 \cdot 6$ & $26: 8$ & $6 \cdot 8$ & $20 \cdot 7$ \\
\hline 0.405 & $32 \cdot 9$ & $26 \cdot 3$ & $6 \cdot 6$ & $20 \cdot 4$ \\
\hline $0 \cdot 30$ & $30 \cdot 3$ & $25 \cdot 0$ & $5 \cdot 3$ & $20 \cdot 1$ \\
\hline $0 \cdot 125$ & $26 \cdot 3$ & $23 \cdot 0$ & $3 \cdot 3$ & $19 \cdot 7$ \\
\hline 0.036 & $23^{\circ} 0$ & $21 \cdot 5$ & 1.5 & $19 \cdot 5$ \\
\hline 0.026 & $21 \cdot 5$ & 20.4 & $1 \cdot 1$ & $19 \cdot 4$ \\
\hline
\end{tabular}

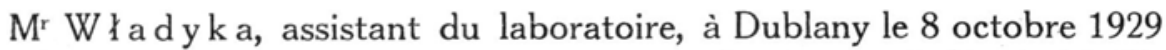
pendant un jour parfaitement clair entre $11^{\mathrm{h}} 35^{\mathrm{m}}$ et $16^{\mathrm{h}} 30^{\mathrm{m}}$ (table II). Évidemment, chaque exemplaire de l'appareil doit être étalonné séparément. Pour les mesures relatives, comme cela se fait fréquemment pendant les études écologiques des associations végétales, l'étalonnage est superflu. 
La représentation graphique des valeurs de la table II (fig. 90) montre que la relation linéaire cesse de valoir pour l'intensité du rayon-

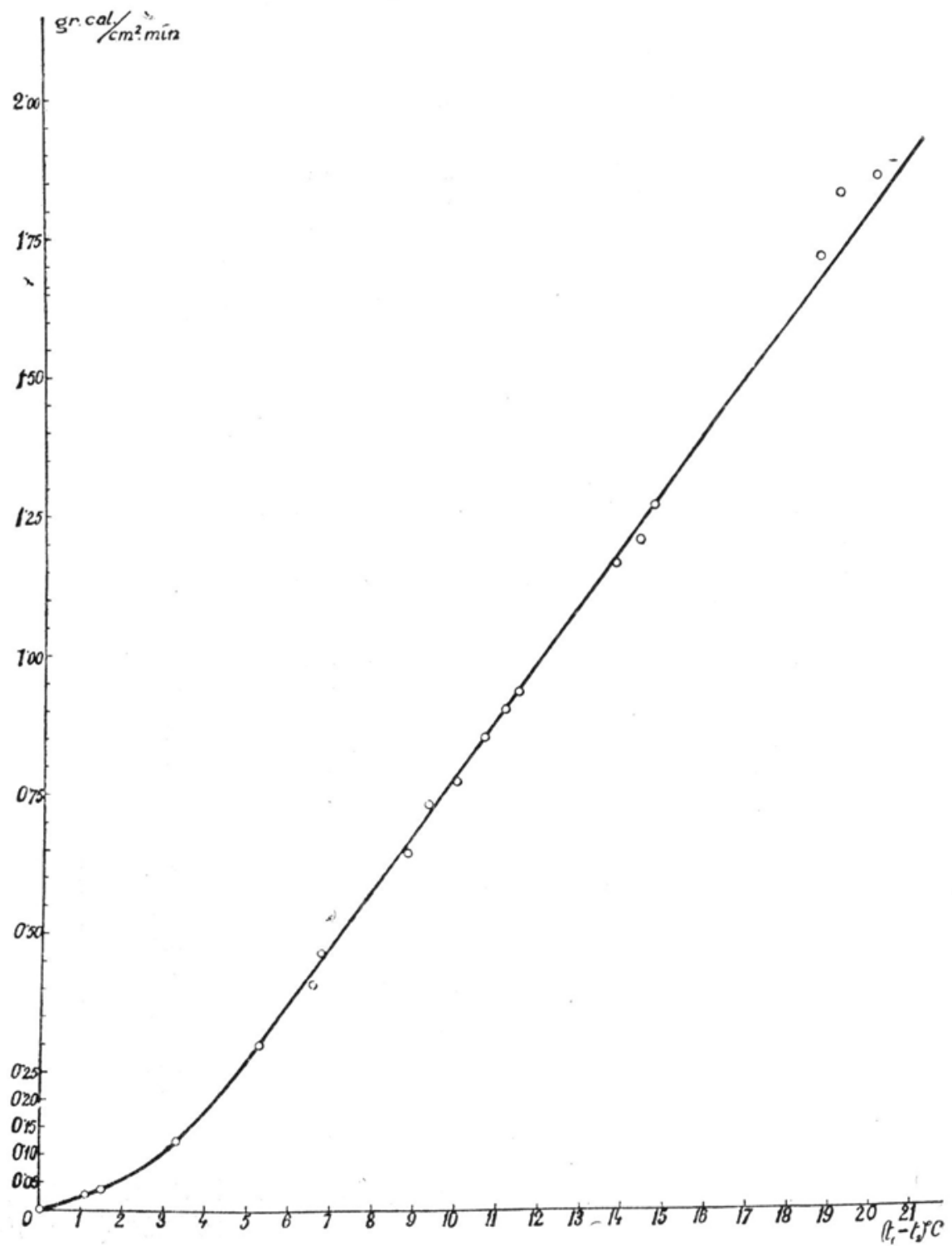

Fig. 90.

nement moindre de $0.25 \mathrm{gr}$. cal./min. $\mathrm{cm}^{2}$ d'après l'échelle suédoise, sur laquelle ont été basées ces observations. La radiation très faible (au- 
dessous de 0.25 ) provoque des différences de la température relativement plus grandes que la radiation plus forte. Les 3 valeurs les plus élevées dévient également de la droite. C'était peut-être l'action de la température de l'air plus élevée. En général, la température de l'air produit une certaine influence sur l'appareil. Cette influence est encore à élucider.

L'appareil que je décrit ici peut être construit à peu de frais. Il est facile à désservir. On pourrait l'employer pour les observations courantes dans les stations météorologiques.

Laboratoire de Botanique de l'École Polytechnique de Lwów. 